\title{
Tuning the Solid-State White Light Emission of Postsynthetic Lanthanide-Encapsulated Double-Layer MOFs for Three-Color Luminescent Thermometry Applications
}

Liya Qiu,${ }^{\dagger}$ Chengfeng Yu, ${ }^{\dagger}$ Xiaoling Wang, ${ }^{\dagger}$ Yangbin Xie,${ }^{\dagger}$ Alexander M. Kirillov Wei Huang, ${ }^{*}, \dagger$ Jipeng Li,${ }^{\dagger}$ Peng Gao, ${ }^{\dagger}$ Ting Wu,${ }^{\dagger}$ Xiangwei Gu, ${ }^{\dagger}$ Qi Nie,${ }^{\dagger}$ and Dayu $\mathrm{Wu}^{*}, \uparrow$

$\dagger$ Jiangsu Key Laboratory of Advanced Catalytic Materials and Technology, Advanced Catalysis and Green Manufacturing Collaborative Innovation Center, School of Petrochemical Engineering, Changzhou University, Changzhou, Jiangsu 213164, China.

¥ Centro de Quimica Estrutural, Instituto Superior Tecnico, Universidade de Lisboa, Av. Rovisco Pais, 1049-001, Lisbon, Portugal. §Peoples’ Friendship University of Russia (RUDN University), 6 Miklukho-Maklaya st., Moscow, 117198, Russian Federation.

Email: wudy@cczu.edu.cn 
(a)

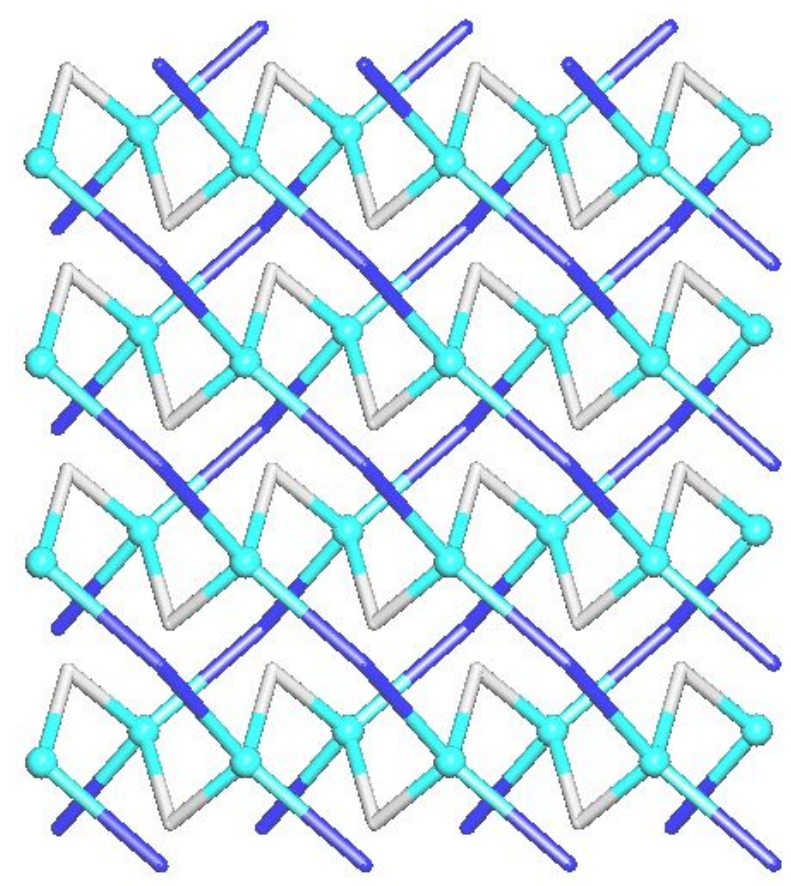

(b)

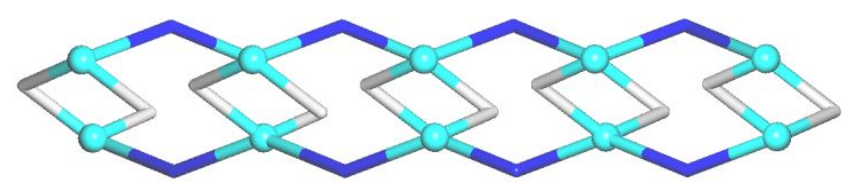

Fig. S1. Front (a) and side topological view (b) of a 2D underlying net in dIMOF showing a 4-connected double layer with the 4L2 topology. Further details: 4-connected Zn1 nodes (cyan balls), centroids of 2-connected $\mu$ - $\mathrm{L}^{2-}$ (gray) and $\mu$-bidpe (blue) linkers. 

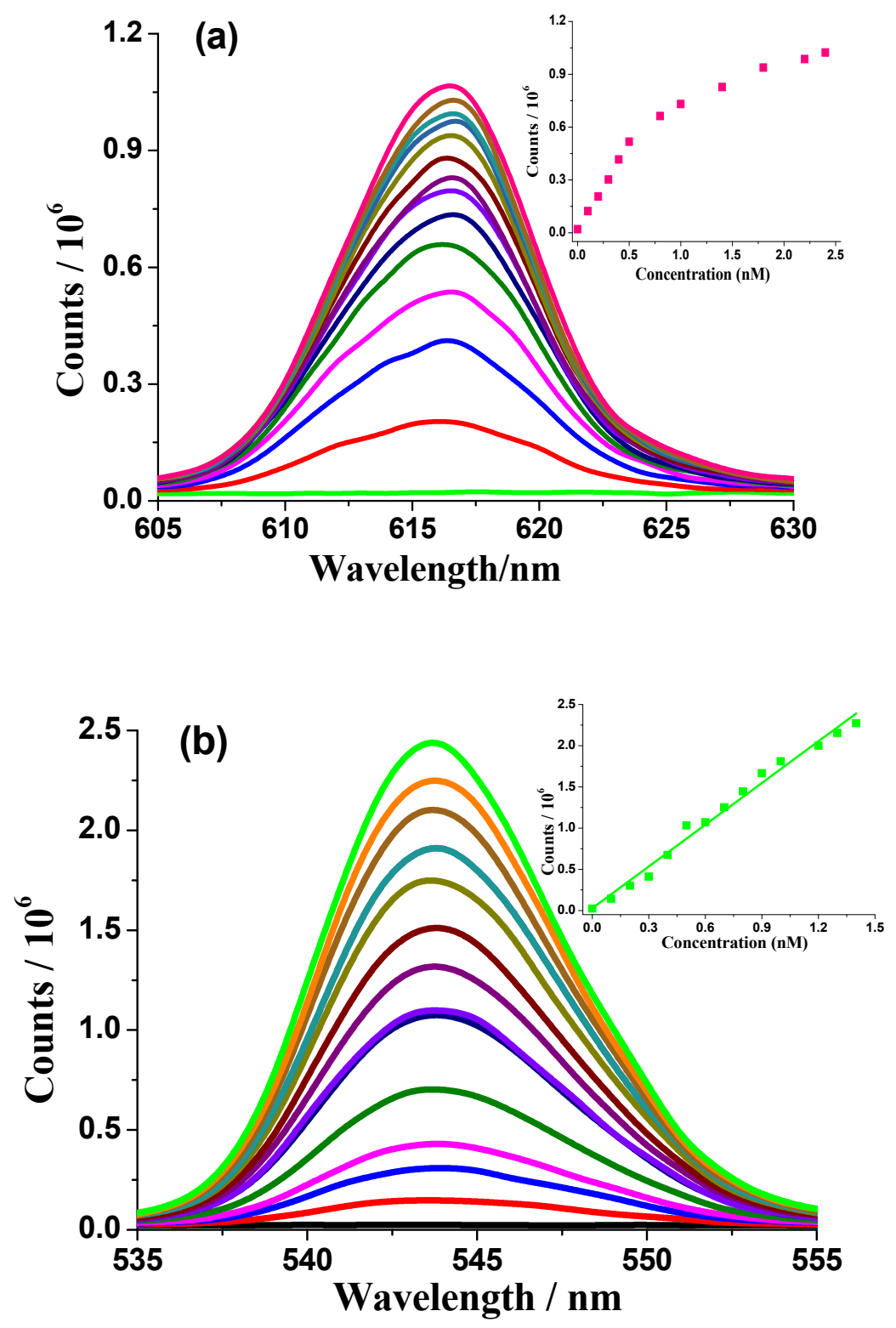

Fig. S2. Fluorescence titration spectra of dIMOF suspension in $\mathrm{CH}_{3} \mathrm{OH}$ in response to nanomolar concentration of $\mathrm{Eu}^{3+}(\mathrm{a})$ and $\mathrm{Tb}^{3+}(\mathrm{b})$. 
(a)

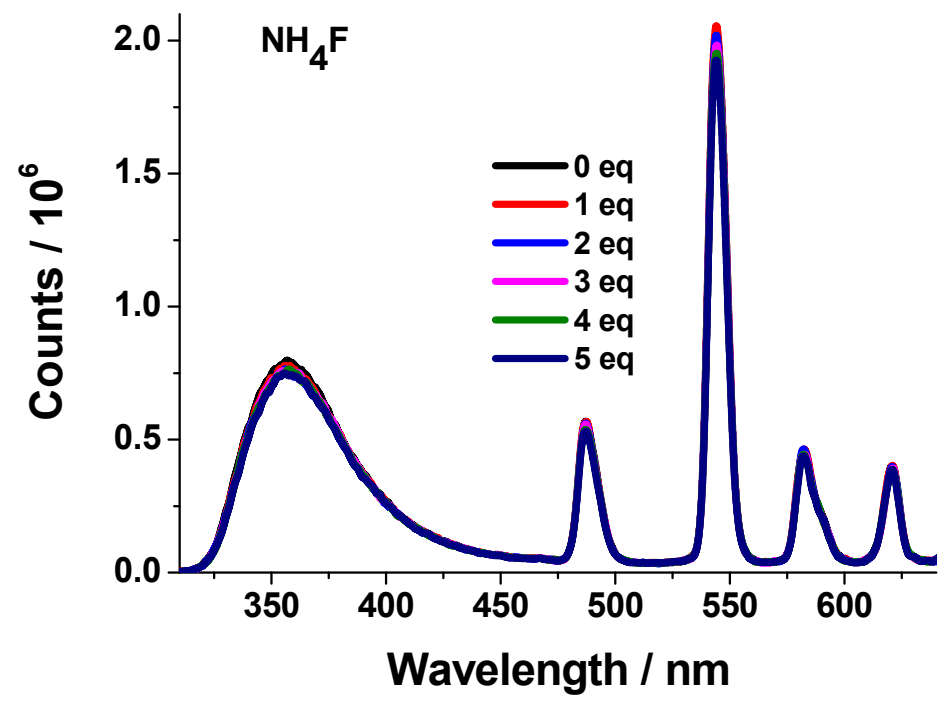

(b)

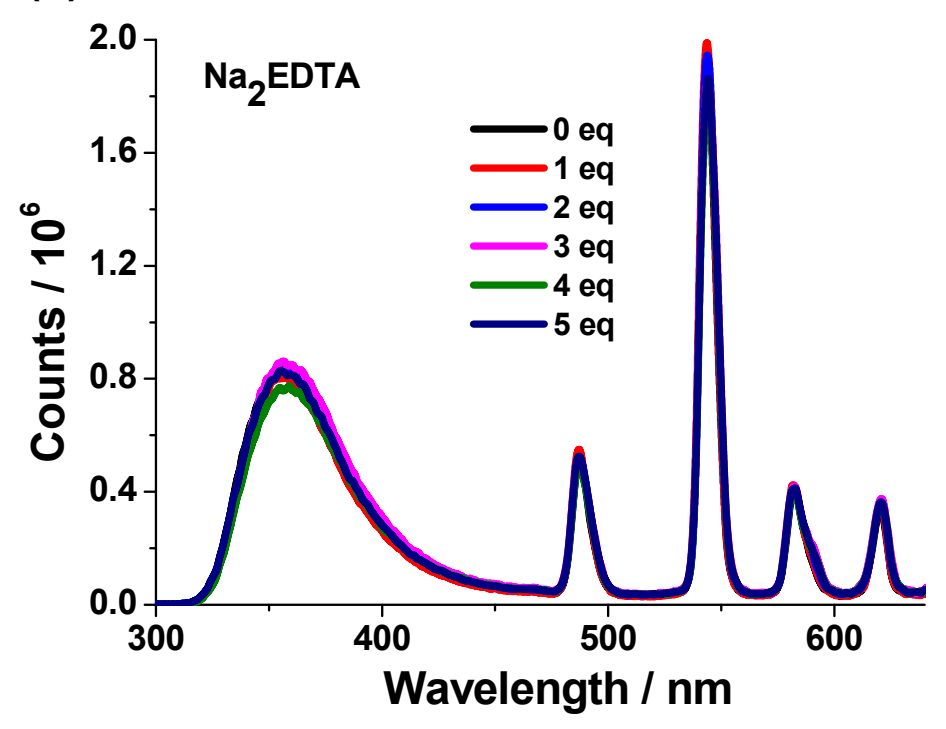

Fig. S3. Fluorescence titration spectra of Tb@dIMOF suspension in $\mathrm{CH}_{3} \mathrm{OH}$ in the presence of the increasing concentration of $\mathrm{NH}_{4} \mathrm{~F}$ (a) and $\mathrm{Na}_{2}$ EDTA (b). 

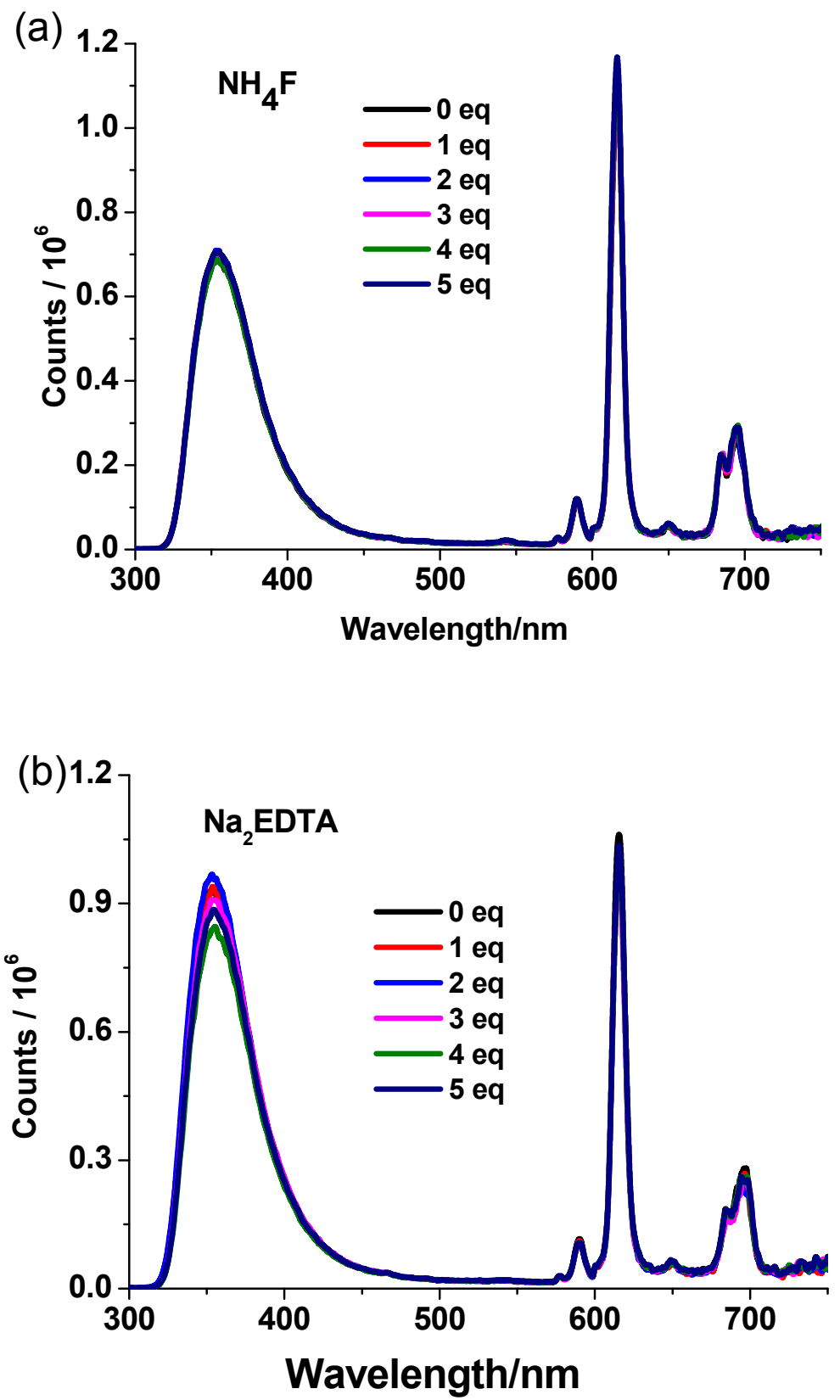

Fig. S4. Fluorescence titration spectra of $\mathbf{E u @ M O F}$ suspension in $\mathrm{CH}_{3} \mathrm{OH}$ in the presence of the increasing concentration of $\mathrm{NH}_{4} \mathrm{~F}$ (a) and $\mathrm{Na}_{2} \mathrm{EDTA}$ (b). 


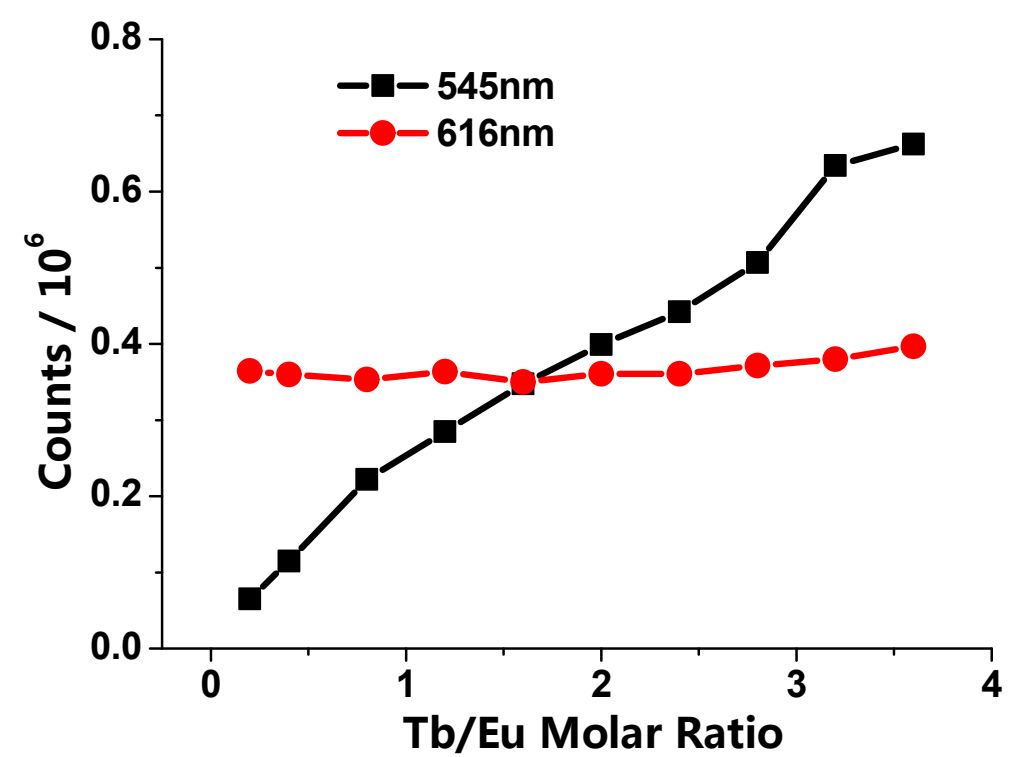

Fig. S5. Job's plot of the green-light $(545 \mathrm{~nm})$ and red-light $(616 \mathrm{~nm})$ emission intensity as a function of the $\mathrm{Tb} / \mathrm{Eu}$ molar ratio. Emission spectra titration with the increasing $\mathrm{Tb} / \mathrm{Eu}$ ratio was undertaken in the suspension of dIMOF $\left(\lambda_{\mathrm{ex}}=270 \mathrm{~nm}\right)$ in methanol. 

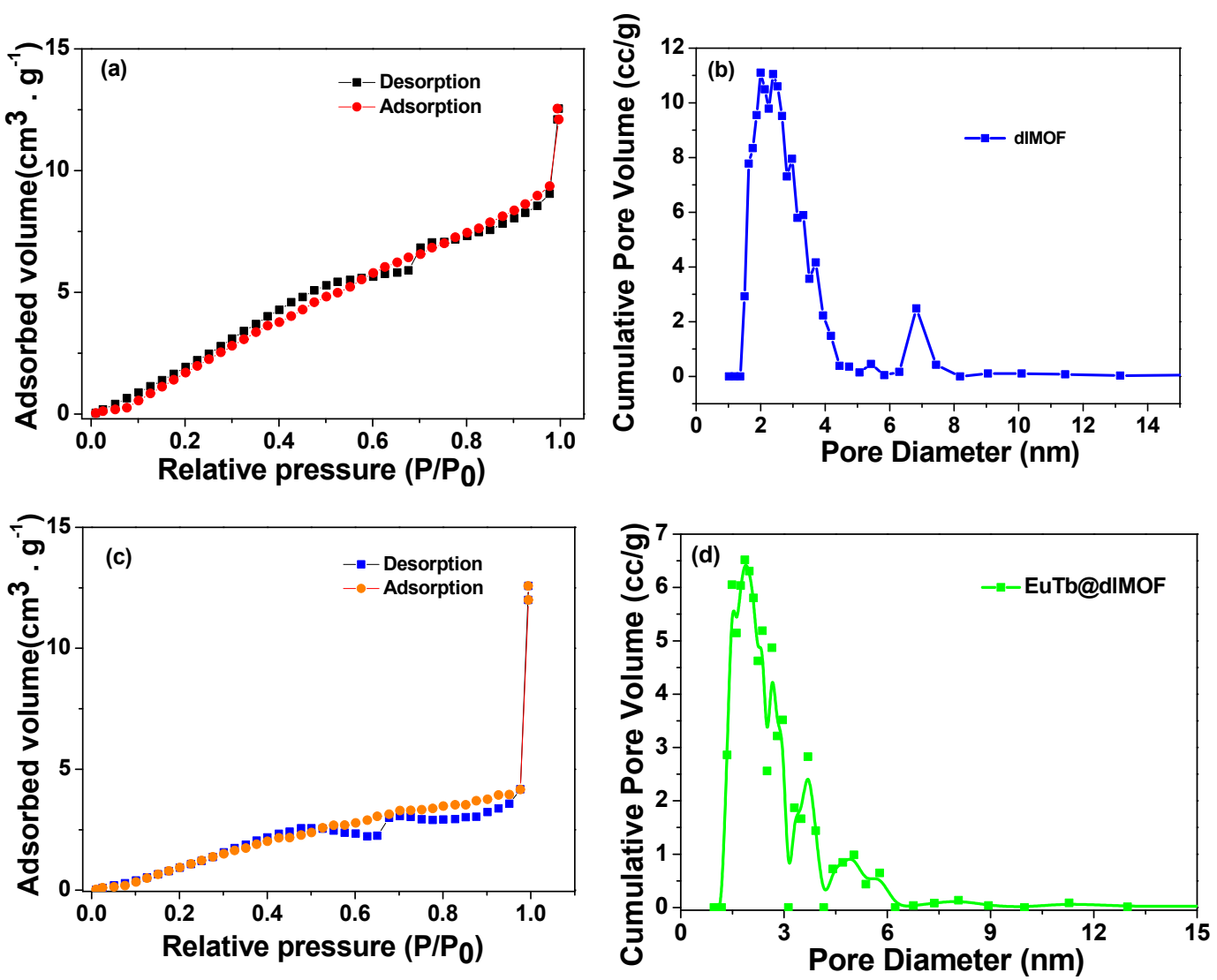

Figure S6. Nitrogen isotherms and pore size distributions of dIMOF $(a, b)$ and EuTb@dIMOF (c,d) with the surface area of 23.5 and $15.3 \mathrm{~m}^{2} / \mathrm{g}$, respectively. 


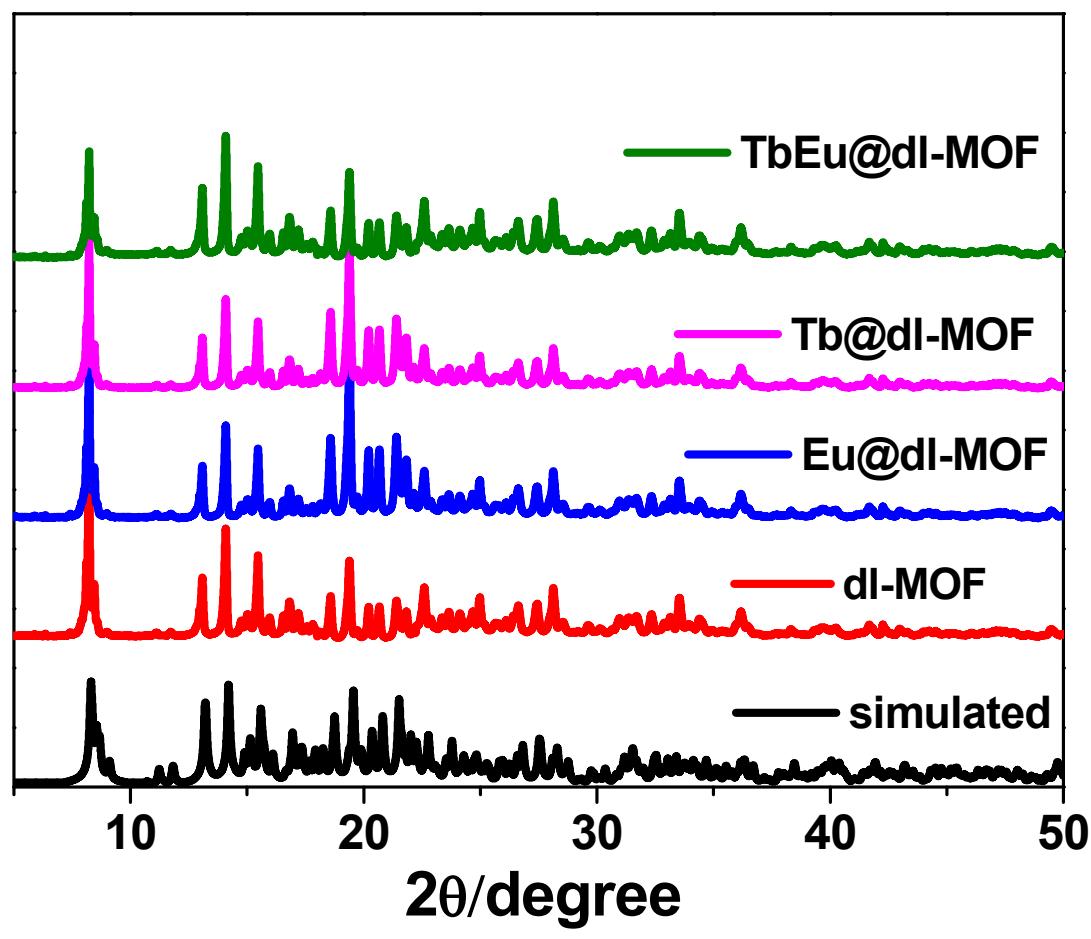

Fig. S7 PXRD patterns of dIMOF and derived $\mathrm{Ln}^{3+}$-loaded MOF materials (Ln@dIMOF; Ln = $\mathrm{Eu}, \mathrm{Tb}$, and $\mathrm{Eu} / \mathrm{Tb})$. 


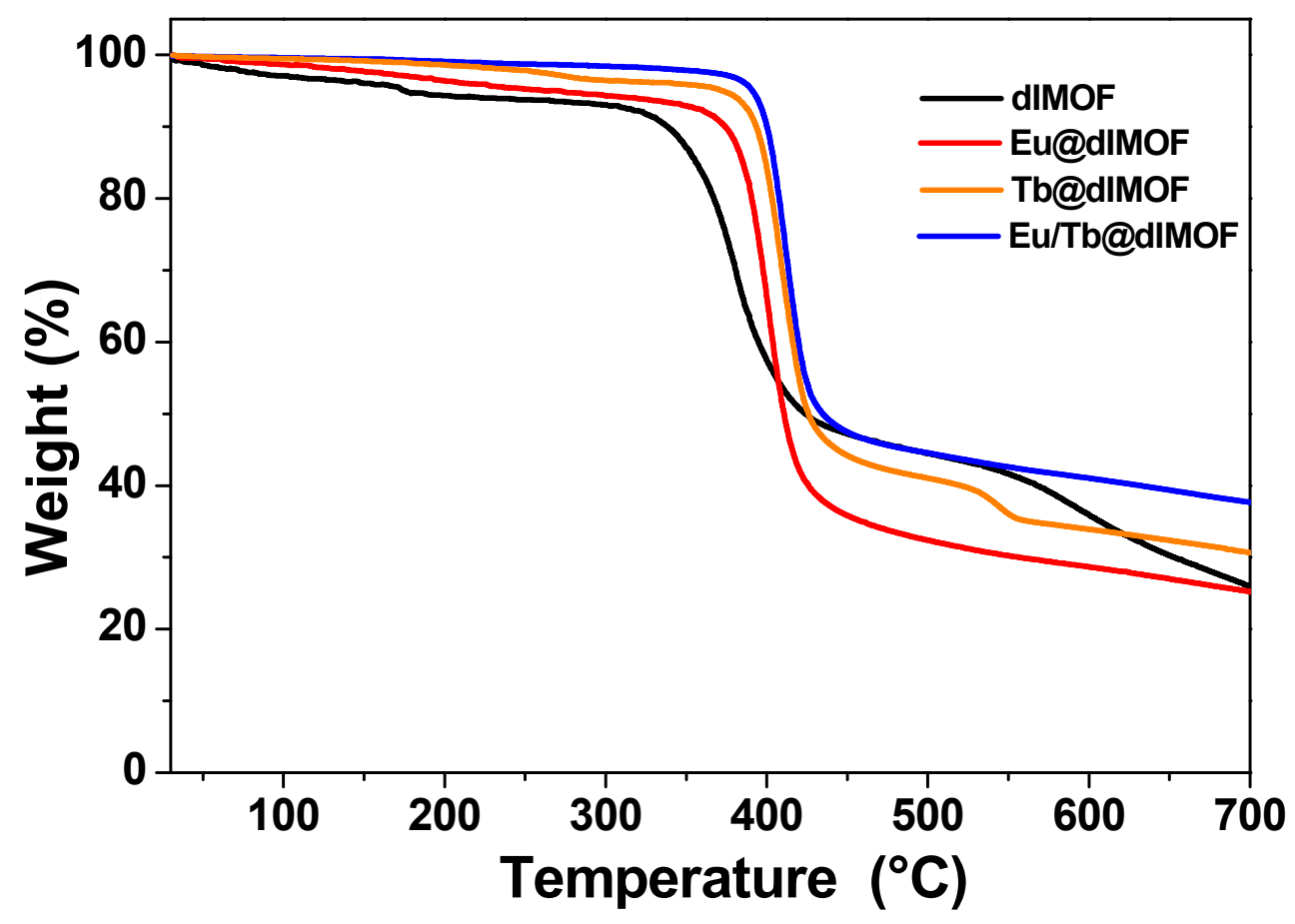

Fig. S8. The thermogravity data of dIMOF, Eu@dIMOF, Tb@dIMOF and EuTb@dIMOF. 


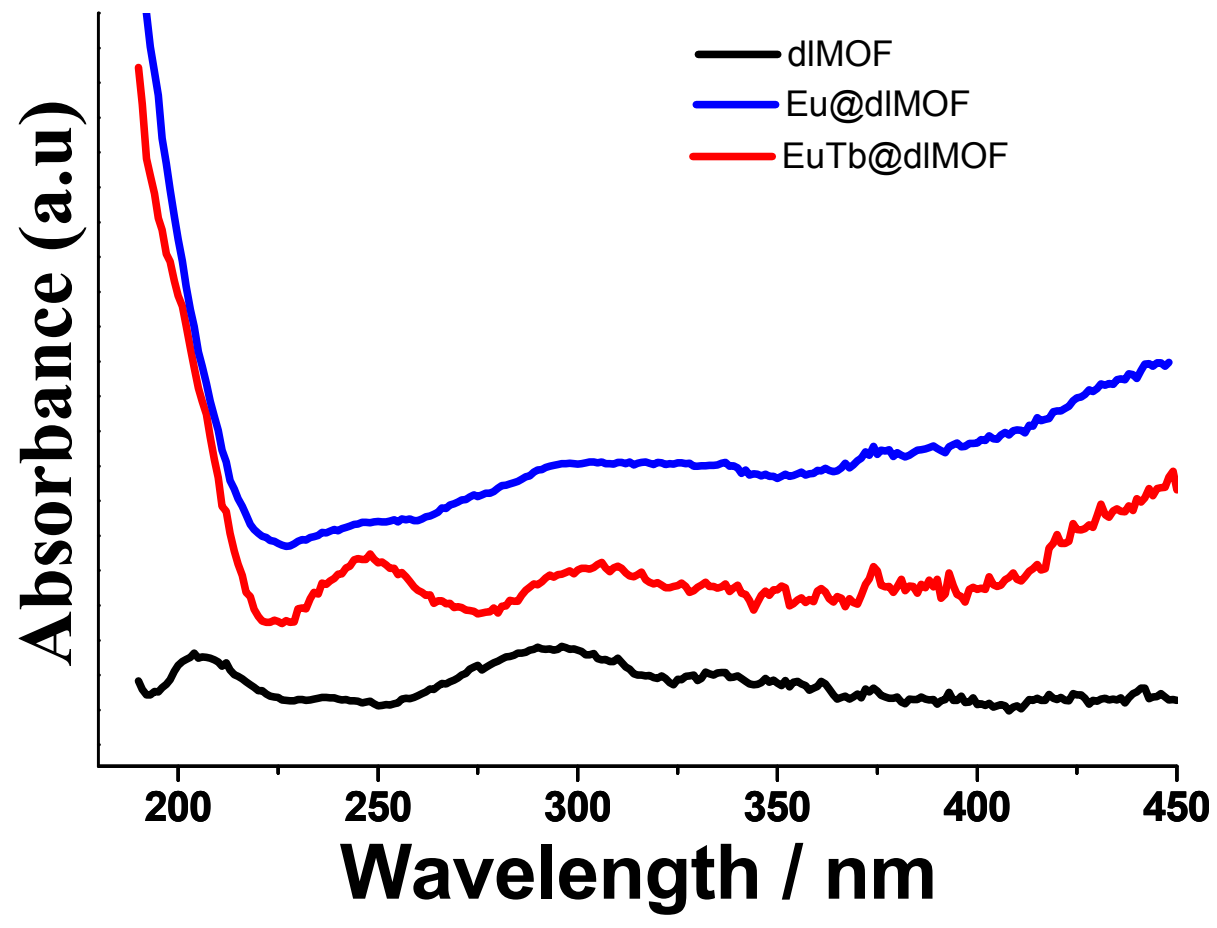

Fig. S9 UV-vis spectra of dIMOF, Eu@dIMOF, and EuTb@dIMOF in solid state. 


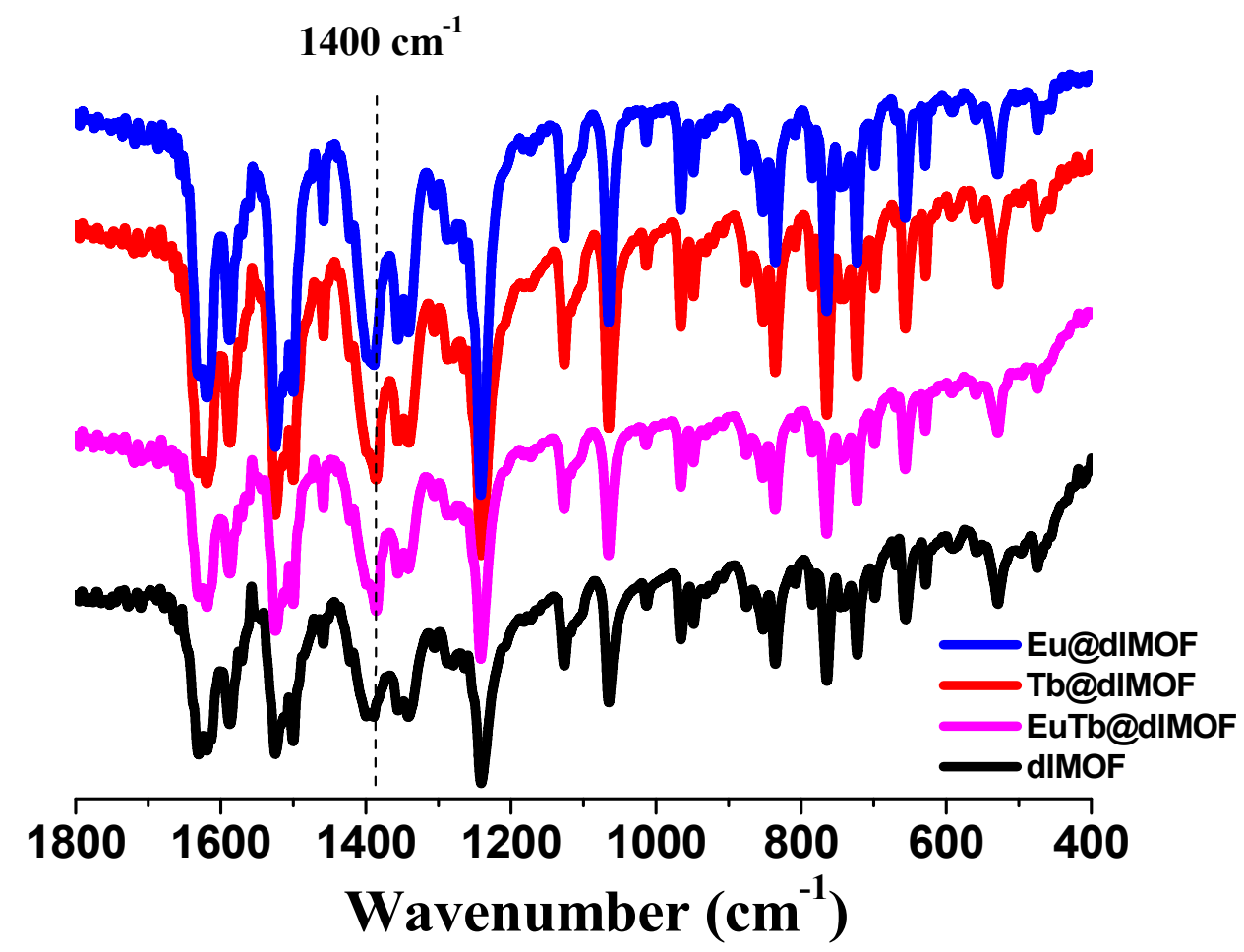

Fig. S10 FT-IR spectra of dIMOF sample and hybrid Ln@dIMOF materials. 
(a)

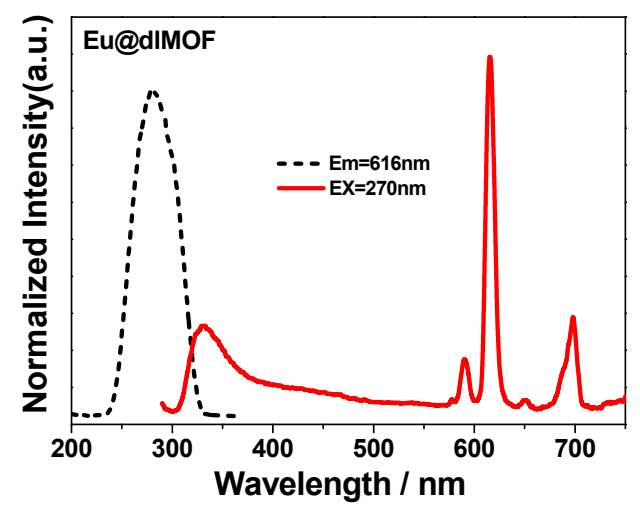

(b)

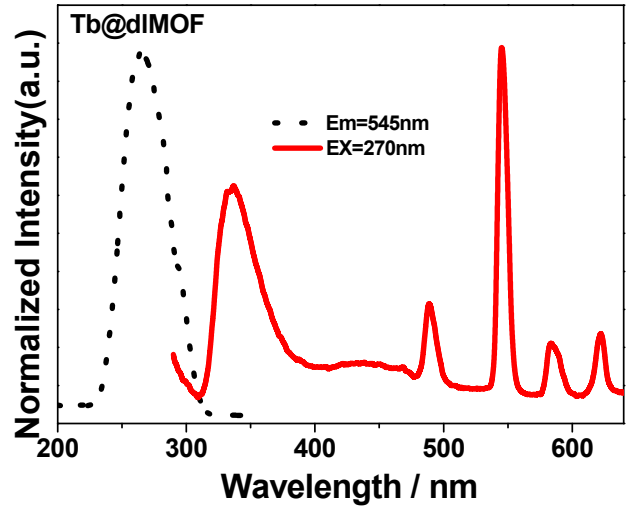

(c)

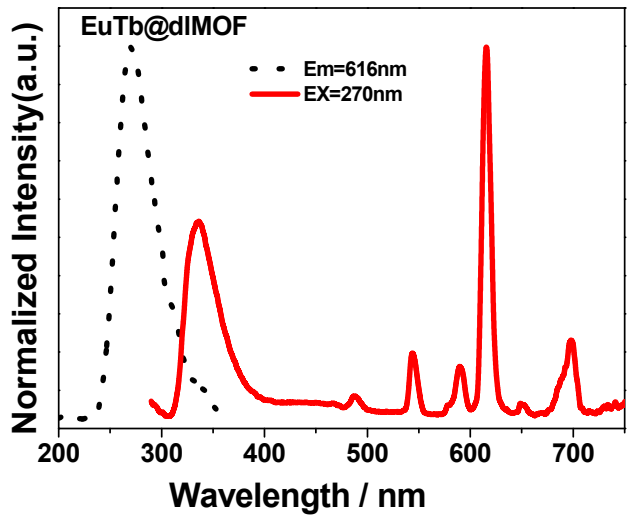

Fig. S11. Solid-state excitation (dotted line) and emission (solid line) spectra of Eu@dIMOF (a), Tb@dIMOF (b), and EuTb@dIMOF (c) at room temperature. All measurements were performed at an excitation wavelength of $270 \mathrm{~nm}$. 


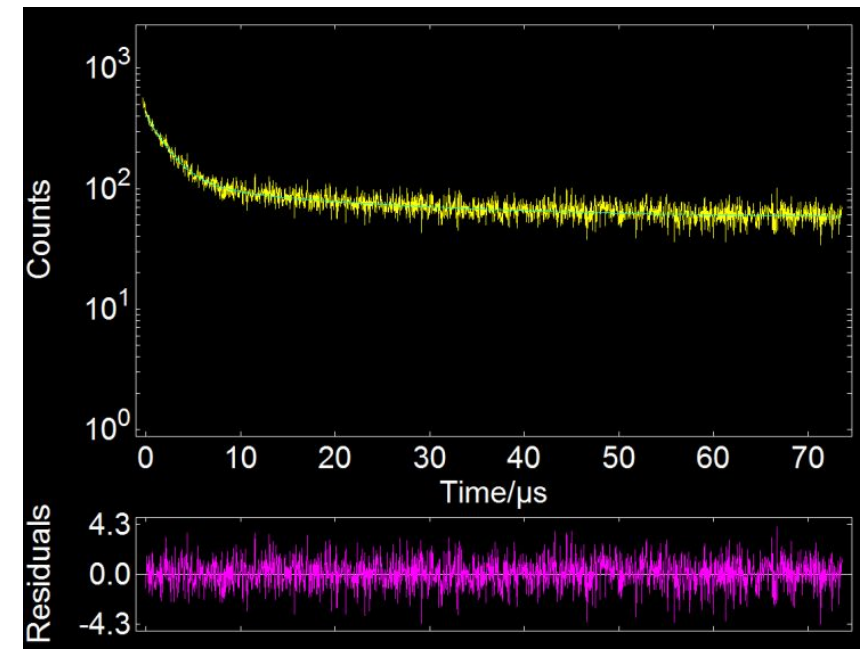

Fig. S12. Relaxation of luminescence signal at $616 \mathrm{~nm}$ with excitation at $270 \mathrm{~nm}$ of Eu@dIMOF solid at room temperature. The blue line is the best least-squares biexponential fit.

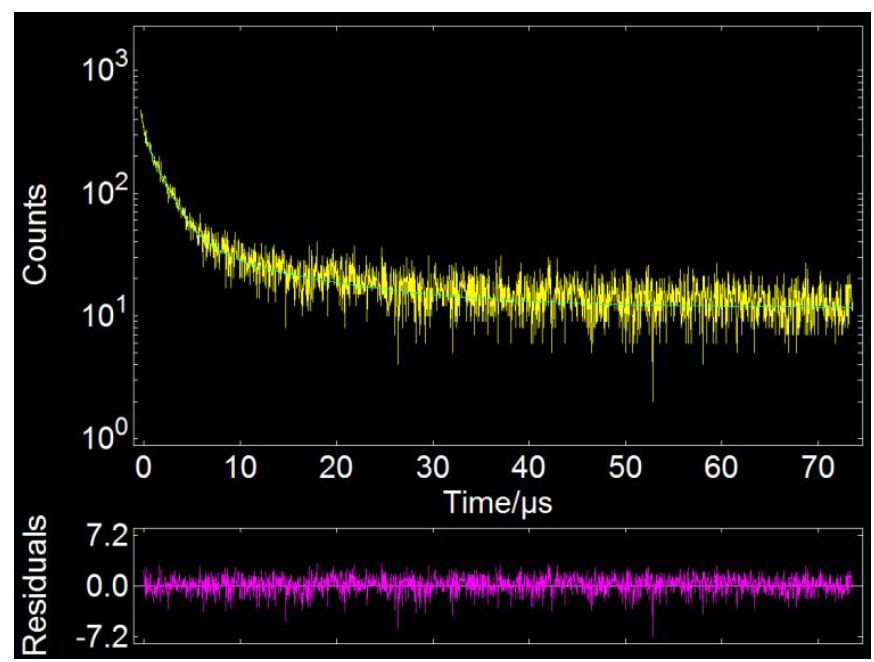

Fig. S13. Relaxation of the luminescence signal at $545 \mathrm{~nm}$ with excitation at $270 \mathrm{~nm}$ of Tb@dIMOF solid at room temperature. The blue line is the best least-squares biexponential fit. 


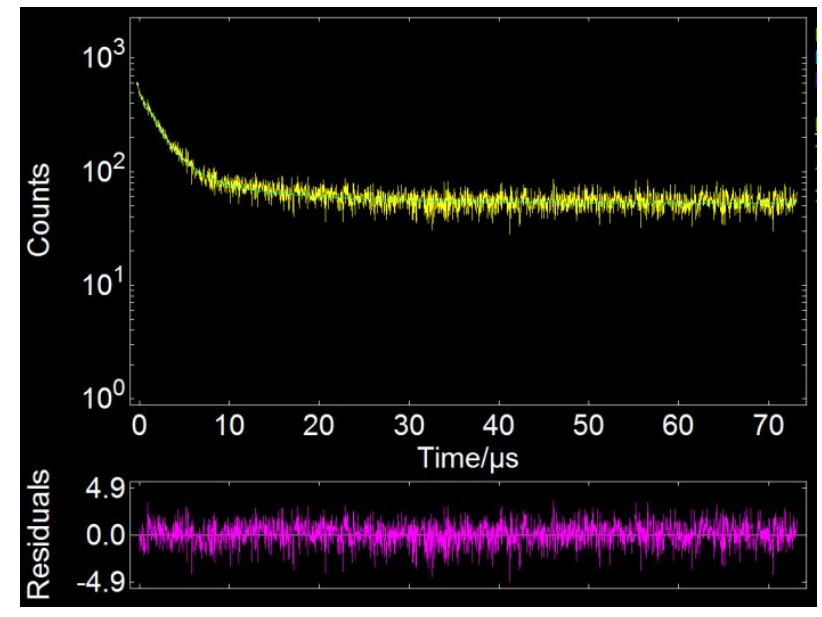

Fig. S14. Relaxation of the $\mathrm{Tb}^{3+}$ at $545 \mathrm{~nm}$ luminescence signal produced by $270 \mathrm{~nm}$ excitation of EuTb@dIMOF solid at room temperature. The blue line is a least-squares biexponential fit.

(a)

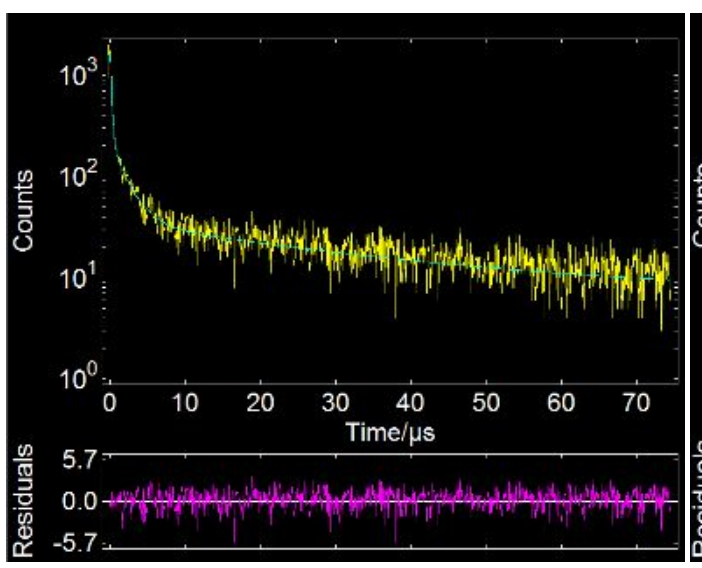

(b)

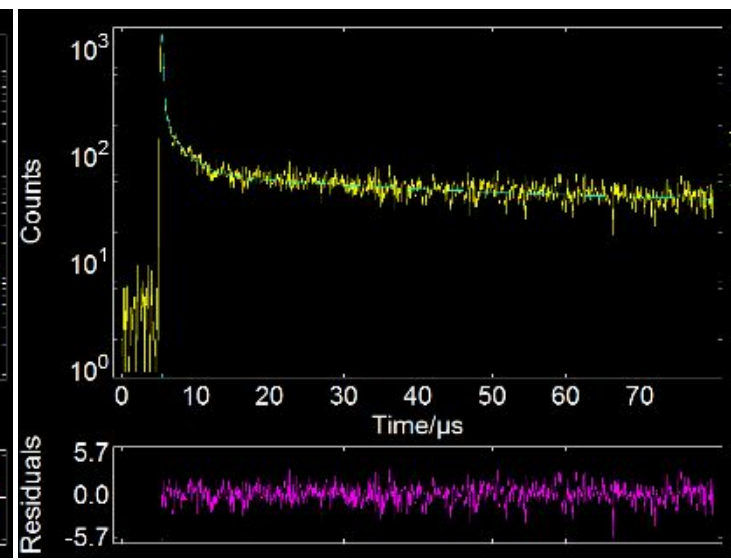

Fig. S15. Low temperature (78 K) relaxation of the luminescence signal at $545 \mathrm{~nm}$ (a) and $616 \mathrm{~nm}$ (b) in the EuTb@dlMOF solid excited at 270 nm. 